\title{
Hyperlipidemia: Management with Proprotein Convertase Subtilisin/Kexin Type 9 (PCSK9) Inhibitors
}

\author{
Muhammed Shabreyar, MD, Salem A. Salem, MD, Mannu Nayyar, MD, \\ Lekha K. George, MD, Nadish Garg, MD, and Santhosh K.G. Koshy, MD, MBA
}

Coronary artery disease is the leading cause of death in United States. Hyperlipidemia is an independent and potentially reversible risk factor for coronary artery disease. The 3-hydroxy-3-methylglutaryl-coenzyme A reductase inhibitors, collectively known as statins, have been the mainstay of pharmacologic therapy. Their availability, ease of administration, low cost, and strong evidence behind safety and efficacy makes them one of the most widely prescribed lipid-lowering agents. However, some patients may be intolerant to statins, and few others suffer from very high serum levels of cholesterol in which statin therapy alone or in combination with other cholesterol-lowering agents is insufficient in reducing serum lipid levels to achieve desired levels. In 2015, the Food and Drug Administration approved a new family of lipid-lowering agents, collectively known as proprotein convertase subtilisin/kexin type 9 (PCSK9) inhibitors.

PCSK9 inhibitors are biologically active molecules that decrease serum low-density lipoprotein cholesterol compared with statin therapy alone. They serve as an alternative to statins for patients who are intolerant to statin or as supplemental therapy in those patients for whom lower levels in serum lowdensity lipoprotein cholesterol are not achieved by statins alone. This article discusses PCSK9 inhibitors, their mechanism of action, indications, efficacy, safety, costs and limitations. ( $\mathrm{J}$ Am Board Fam Med 2018;31:628-634.)

Keywords: Cholesterol, Coronary Artery Disease, Hypolipidemic Agents

Cardiovascular disease is the leading cause of death in the United States, with coronary artery disease being the most common cause of heart disease. Increased serum low-density lipoprotein cholesterol (LDL-C) concentration is considered one of

This article was externally peer reviewed.

Submitted 24 November 2017; revised 17 January 2018; accepted 21 January 2018.

From Division of Cardiology, Department of Medicine, University of Tennessee Health Sciences Center, Memphis, TN (MS, SAS, MN, NG, SKGK); Division of Nephrology, Department of Medicine, University of Tennessee Health Sciences Center, Memphis (LKG); Department of Physician Assistant Studies, University of Tennessee Health Sciences Center, Memphis (LKG); Regional One Health, Memphis (SKGK).

Funding: none.

Conflict of interest: none declared.

Corresponding author: Santhosh K.G. Koshy, MD, MBA, Professor of Medicine and Vice Chairman, Department of Medicine, University of Tennessee Health Sciences Center, 956 Court Avenue, Suite A312, Memphis, TN 38163 (Email: skoshy@uthsc.edu). the major risk factors associated with coronary artery disease ${ }^{1}$, and, consequently, a reduction in LDL-C levels has been shown to significantly reduce the risk of major cardiovascular events. ${ }^{2}$ Traditionally, statins have been the cornerstone of cholesterol-lowering therapy, demonstrating a reduction in cardiovascular disease mortality and morbidity in several clinical trials. ${ }^{3}$ In the majority of patients, statins have been very effective in lowering LDL-C; however there remains a subset of patients in whom LDL-C reduction is important, but they are either intolerant to statin therapy or in whom statin therapy alone is inadequate at lowering LDL-C concentration, such as those with familial hypercholesterolemia. ${ }^{4}$ These patients, even with the most efficacious lipid-lowering therapy, have substantial risks of atherosclerotic cardiovascular disease (ASCVD) and acute coronary and vascular events. Recently, proprotein convertase subtilisin/kexin type 9 (PCSK9) inhibitors have 


PCSK9, a protein (protease) secreted primarily by liver, binds to LDL receptors on hepatocytes and
promotes internalization and lysosomal degradation of LDL receptors, thereby reducing the
number of LDL receptors. Reduction in LDL receptors result in elevated serum LDL-C levels.
PCSK9 inhibitors are monoclonal antibodies against PCSK9, thereby preventing internalization and
lysosomal degradation of LDL receptors. This increases LDL receptor density, thereby reducing
serum LDL-C levels.
Evolocumab and alirocumab are two commercially available PCSK9 inhibitors that are FDA
approved for human use.
Available products
Both evolocumab and alirocumab are administered as subcutaneous injection once a month or every
2 weeks
Should be stored in refrigerator and warmed to room temperature before use.

PCSK9, proprotein convertase subtilisin/kexin type 9; LDL-C, low-density lipoprotein cholesterol; FDA, Federal Drug Administration.

emerged as very effective agents for reducing LDL-C and have the potential to improve risks for death and major adverse cardiovascular events in certain patients who are at high risk for ASCVD and acute events. In this review, we discuss the mechanism of action of PCSK9 inhibitors, their safety, and present cost and summarize current data on their efficacy in different patient populations.

\section{Role of PCSK9 in Cholesterol Metabolism}

Multiple studies demonstrating a linear relationship between lowering serum LDL-C levels and improving outcomes in cardiovascular disease ${ }^{5}$, along with inadequacy of statin with or without concomitant other lipid-lowering strategies to reduce LDL-C to desired levels, have sparked interest in developing novel therapies for LDL-C reduction. PCSK9 is a protein belonging to a family of proteases called proprotein convertases, which is secreted mostly by the liver, with small amounts secreted from the small intestines and the kidneys. It is encoded by the PCSK9 gene located on chromosome 1p32.3. ${ }^{6}$ Under normal conditions, PCSK9 promotes hypercholesterolemia by binding to LDL receptors on the surface of hepatocytes, following which, the LDL receptor is internalized and transported to lysosomes for degradation. This leads to a decrease in the number of LDL receptors on the hepatocyte surface and, therefore, resulting in reduced clearance of LDL-C from the blood ${ }^{7}$, resulting in elevated serum LDL-C levels. There is also supporting data that suggests the low-density lipoprotein receptor and apolipoprotein E2 receptor are partly regulated by PCSK9. ${ }^{6}$

PCSK9 was first recognized as a potential target for LCL-C-lowering therapy in 2003. ${ }^{8,9}$ Since then, genetic studies of patients have confirmed the role of PCSK9 in LDL-C regulation. A gain of function mutation in the PCSK9 gene has been recognized as a cause of a rare but severe form of autosomal dominant hypercholesterolemia. ${ }^{9}$ Conversely, a loss of function mutation in the PCSK9 gene has been associated with significantly reduced levels of LDL-C and lower risk of coronary heart disease. ${ }^{10}$ These observations have led to PCSK9 being recognized as a potential target for modulating cholesterol metabolism.

\section{PCSK9 Inhibitors}

The goal of PCSK9 inhibitor therapy is to reduce serum LDL-C levels by curtailing PCSK9 activity. Suppressing PCSK9 activity leads to decreased LDL receptor degradation, therefore, increasing LDL receptor expression on the hepatocyte surface and resulting in increased clearance of LDL-C from the blood stream (Table 1). Although other therapeutic approaches to inhibit PCSK9 by using compact proteins called adnectins and short interfering RNA targeting PCSK9 synthesis in hepatocyte have been developed, as of now, antiPCSK9 monoclonal antibodies remain the mainstay PCSK9 inhibitor therapy. The Food and Drug Administration in 2015 approved evolocumab (Repatha) and alirocumab (Praluent) for use in patients with familial hypercholesterolemia and patients with cardiovascular disease who require further reduction in LDL-C. Both of these agents are fully humanized monoclonal antibodies that need to be administered via subcutaneous injections. ${ }^{11}$ Both evolocumab and alirocumab are approved for administration once a month or every 2 weeks. The drug is stored in a refrigerator and warmed to room temperature before administration. ${ }^{11}$ Before administration, both evolocumab 
Table 2. Effects of Treatment with PCSK9 Inhibitors

\begin{tabular}{|c|c|}
\hline LDL-C reduction & $\begin{array}{l}\text { Treatment with evolocumab or alirocumab results in approximately } 55 \% \text { or } 53 \% \text { reduction in } \\
\text { LDL-C levels, respectively. }\end{array}$ \\
\hline Effect on lipid panel & $\begin{array}{l}\text { Evolocumab and alirocumab results in } 7.6 \% \text { and } 8 \% \text { increases in HDL-C, respectively. There } \\
\text { was reduction in non-HDL-C, total cholesterol, triglycerides, and lipoprotein(a) levels. }\end{array}$ \\
\hline Effect on clinical outcomes & $\begin{array}{l}\text { PCSK9 inhibitor results in } 15 \% \text { to } 48 \% \text { lower hazard of a composite of CV death, MI, stroke, } \\
\text { hospitalization for UA, or coronary revascularization (1.5\% absolute reduction of events) } \\
\text { compared with standard medical therapy. Long-term outcome data are not available. }\end{array}$ \\
\hline Plaque regression & $\begin{array}{l}\text { Significantly more plaque volume reduction seen with PCSK9 inhibitor treatment as compared } \\
\text { with statin therapy. }\end{array}$ \\
\hline
\end{tabular}

PCSK9, proprotein convertase subtilisin/kexin type 9; LDL-C, low-density lipoprotein cholesterol; HDL-C, high-density lipoprotein cholesterol; CV, cardiovascular; MI, myocardial infarction; UA, unstable angina.

and alirocumab should be allowed to warm for at least 30 minutes at room temperature. Refrigeration can be avoided if the drug can be stored in original carton at $77^{\circ} \mathrm{F}$ (or $25^{\circ} \mathrm{C}$ ) and used within 30 days.

\section{Efficacy of Monoclonal Antibodies to PCSK9 and Their Impact on Cardiovascular Outcomes}

Monoclonal antibodies have been very effective in lowering serum LDL-C. A well-powered metaanalysis by Zhang XL et al ${ }^{12}$ including 25 randomized controlled trials encompassing 12,200 patients evaluated the efficacy and safety profile of monoclonal antibodies to PCSK9. Monthly treatment with $420 \mathrm{mg}$ of evolocumab reduced LDL-C by $55 \%$ compared with placebo and by $36 \%$ compared with ezetimibe. Furthermore, it increased highdensity lipoprotein cholesterol (HDL-C) by $7.6 \%$ compared with placebo and by $6.4 \%$ versus ezetimibe. Similarly, biweekly treatment with alirocumab decreased LDL-C by $53 \%$ and $30 \%$ versus placebo and ezetimibe, respectively, while increasing HDL-C by $8 \%$ compared with placebo (Table 2).

The maximal reduction in LDL-C reaches a plateau once all the PCSK9 is bound, with additional dosing only prolonging duration of action. ${ }^{11}$ In addition to having a positive effect on the LDL-C and HDL-C profile, a pooled analysis of data from 3 12-week trials found evolucumab reduced non-HDL-C by $58 \%$, total cholesterol by $40 \%$, triglycerides by $17 \%$, and lipoprotein(a) by $29 \% .{ }^{13}$ Efficacy of evolucumab was comparable in both diabetics and nondiabetics. ${ }^{13}$ In a separate prospective trial, alirocumab was found to have similar effects on the overall lipid profile in both diabetics and nondiabetics. ${ }^{11}$ The lipoprotein(a)- reducing effect of PCK9 monoclonal antibodies ${ }^{11,14}$ seems to be an additional benefit over statins. Lipoprotein(a) has been recognized as an independent risk factor for cardiovascular disease in epidemiologic studies. ${ }^{15}$

There is accumulating evidence that PCSK9 monoclonal antibodies are very effective at reducing LDL-C levels. ${ }^{11,16}$ OSLER 1 and OSLER 2 were 2 open-labeled randomized trials that looked at the safety and efficacy of evolocumab. These trials showed that evolocumab reduced the level of LDL cholesterol by $61 \%$, from a median of $120 \mathrm{mg}$ per deciliter to $48 \mathrm{mg}$ per deciliter $(P$ value $<$ $.001){ }^{17}$ After 1 year of treatment, adverse event rates were similar in both groups, although the evolocumab group had more neurocognitive events reported. ${ }^{17}$ Recently, the FOURIER trial showed a $59 \%$ reduction in LDL-C levels that was found at 4 weeks and persisted throughout the mean follow-up of 2.2 years when evolocumab was added to statin therapy. It also showed that evolocumab treatment, in comparison to standard medical therapy, significantly reduced the risk of the primary end point (composite of cardiovascular death, myocardial infarction, stroke, hospitalization for unstable angina, or coronary revascularization) by $1.5 \%$ $(9.8 \%$ versus $11.3 \%)$, with a hazard ratio of 0.85 (95\% CI, 0.79-0.92; $P<.001) .{ }^{18}$ There was also reduction in the key secondary end point of composite of cardiovascular death, myocardial infarction, or stroke by $1.5 \%$ (5.9\% versus $7.4 \%)$, with a hazard ratio of 0.80 (95\% CI, 0.73-0.88; $P<$ .001). ${ }^{18}$ With the exception of injection site reactions, evolocumab did not increase the rate of serious adverse events, including new-onset diabetes and neurocognitive events. Similarly, the trial Global Assessment of Plaque Regression with a PCSK9 Antibody as Measured by Intravascular Ul- 
Table 3. PCSK9 Inhibitors: Safety Profile and Adverse Reactions

\begin{tabular}{|c|c|c|}
\hline Safety Profile & Evolocumab & Alirocumab \\
\hline $\begin{array}{l}\text { Common side } \\
\text { effects }\end{array}$ & $\begin{array}{l}\text { Upper respiratory tract infection, influenza, } \\
\text { gastroenteritis, nasopharyngitis, injection site } \\
\text { reactions. }\end{array}$ & $\begin{array}{l}\text { Upper respiratory tract infection, influenza, } \\
\text { nasopharyngitis, gastroenteritis, injection site } \\
\text { reactions. }\end{array}$ \\
\hline Pregnancy & $\begin{array}{l}\text { No data available on use in pregnant women to } \\
\text { inform a drug-associated risk. }\end{array}$ & $\begin{array}{l}\text { No available data on use in pregnant women to inform } \\
\text { a drug-associated risk. }\end{array}$ \\
\hline Lactation & $\begin{array}{l}\text { No information on its presence in human milk, } \\
\text { effects on the breastfed infant, or on milk } \\
\text { production. }\end{array}$ & $\begin{array}{l}\text { No information on its presence in human milk, effects } \\
\text { on the breastfed infant, or on milk production. }\end{array}$ \\
\hline Pediatric use & $\begin{array}{l}\text { The safety profile of Repatha in these adolescents } \\
\text { was similar to that described for adult patients } \\
\text { with HoFH. Safety and efficacy not established } \\
\text { in HoFH patients }<13 \text { years with HoFH and } \\
\text { in pediatric patients with primary } \\
\text { hyperlipidemia or HeFH. }\end{array}$ & $\begin{array}{l}\text { Safety and efficacy in pediatric patients have not been } \\
\text { established. }\end{array}$ \\
\hline Geriatric use & Safety profile similar to younger adults. & Safety profile similar to younger adults. \\
\hline Renal impairment & No dose adjustment is needed. & $\begin{array}{l}\text { No dose adjustment is needed for mild or moderately } \\
\text { impaired renal function. No data are available in } \\
\text { severe renal impairment. }\end{array}$ \\
\hline Hepatic impairment & $\begin{array}{l}\text { No dose adjustment in mild to moderate hepatic } \\
\text { impairment (Child-Pugh A or B). No data are } \\
\text { available in severe impairment. }\end{array}$ & $\begin{array}{l}\text { No dose adjustment in mild to moderate hepatic } \\
\text { impairment. No data are available in severe } \\
\text { impairment. }\end{array}$ \\
\hline
\end{tabular}

PCSK9, proprotein convertase subtilisin/kexin type 9; HoFH, homozygous familial hypercholesterolemia; HeFH, heterozygous familial hypercholesterolemia.

trasound demonstrated an improvement in coronary plaque volume in patients given evolocumab in addition to statin therapy. ${ }^{19}$

We await the ODYSSEY OUTCOMES ${ }^{20}$ trial, which evaluates the effect of adding alirocumab in cardiovascular outcomes in patients after an acute coronary event. In a post hoc analysis of a 78-week trial comparing alirocumab with placebo in 2341 patients at high risk for cardiovascular events (ODYSSEY LONG TERM trial), in addition to significant reduction of LDL-C levels, the rate of major cardiovascular events (death from coronary heart disease, nonfatal myocardial infarction, fatal or nonfatal ischemic stroke, or unstable angina requiring hospitalization) was found to be lower $(1.7 \%$ versus $3.3 \%$; hazard ratio, $0.52 ; 95 \%$ CI, 0.31-0.90; nominal $P=.02$ ) among patients who received alirocumab in addition to statin. ${ }^{16}$

\section{Safety Profile of the Monoclonal Antibodies to PCSK9}

With their profound LDL-C-lowering effects, PCSK9 inhibitors offer both patients and clinicians a potent alternative or adjunct to current therapy, and their safety profile is as impressive. When comparing emergent adverse events, the overall incidence of these events is not significantly different in placebo, ezetimibe, or PCSK9 inhibitor patient populations. ${ }^{12}$ Tolerability is quite high, with low rates of discontinuation in long-term studies of the fully human monoclonal antibodies, alirocumab and evolocumab (Table 3). There are no known drug interactions for either of the medications.

Alirocumab was associated with an increased rate of injection-site reactions (relative risk 1.48, $6 \%$ ) and with a lower risk of death when compared with placebo at 52 weeks. ${ }^{12,20}$ Patients receiving evolocumab had injection-site reactions occurring in $2.1 \%$ compared with $1.6 \%$ in the placebo arm, with $90 \%$ of these reactions classified as mild. ${ }^{21}$ Rates of allergic reactions did not differ significantly between placebo and evolocumab patients. ${ }^{21}$ No difference in reported adverse events was found between the month and bimonthly administration of evolocumab at 12 weeks.

There has been no significantly identifiable trend in the incidence of musculoskeletal or connective tissue disorders, creatine kinase elevation, influenza, nasopharyngitis or autoantibody development reported with the fully human monoclonal antibodies. $^{12,20}$

There has been some concern with respect to adverse neurocognitive events; however, they seem unlikely given the size and inability of these agents to cross the blood-brain barrier. Nonetheless, in the EBBINGHAUS substudy of the recently con- 
cluded FOURIER study, neurocognitive function (to include memory, attention, and reaction time) was assessed and evolocumab was found to be no different from placebo by noninferiority analysis. ${ }^{22}$

\section{Clinical Implications}

Increased serum LDL-C concentration has always been considered a major risk factor associated with ASCVD. Although lifestyle modification has an important role in lowering LDL-C levels, pharmacotherapy is often needed to further reduce LDL-C levels, particularly in patients with ASCVD. Statins, also known as 3-hydroxy-3-methylglutaryl-coenzyme A reductase inhibitors, are the most commonly prescribed pharmacologic agents for patients with ASCVD. Although role of statin therapy has been established in both primary prevention as well as progression of $\mathrm{ASCVD}^{23}$, it is associated with clinically significant side effects that include increased risk of diabetes, muscle pain, and damage to hepatocytes. ${ }^{24}$ Although their frequency is low, these side effects are often the reason why statin therapy is not tolerated by some patients. Moreover, a subset of patients, whose genomic profile predisposes them to very high levels of LDL-C due to alteration in LDL-C metabolism, will not achieve the desired therapeutic effect from statin therapy alone or in combination with other lipid-lowering agents.

The need for a more potent LDL-C-lowering therapy, called for more extensive research geared toward mechanisms of LDL-C clearance from the serum. PCSK9 proteins were recognized as important modulators in lipid metabolism. Over the past decade, considerable effort has been made to target PCSK9 to lower serum LDL-C levels. ${ }^{25}$ In 2015, the Food and Drug Administration approved 2 new humanized monoclonal antibodies, evolocumab (Repatha) and alirocumab (Praluent). These 2 new agents are attractive therapeutic modalities for practitioners treating difficult dyslipidemia, who otherwise have limited options. Moreover, infrequent subcutaneous administration (once every 2 weeks to 4 weeks) guarantees improved compliance and steady blood levels in comparison to medications taken orally.

Although PCSK9 inhibitors seem to take the battle against hyperlipidemia to a whole new level, these novel therapeutic options have a long way to go to gain popularity among health care practitioners. Limited by their annual costs of $\$ 14,000$ to $\$ 15,000$,
PCSK9 inhibitors add extra costs to the health care system. When compared with the annual costs of statin therapy (assumed at $\$ 485$ based on low-cost generic therapy), PCSK9 inhibitors are clearly much more expensive. It is not clear if this extra cost is justified by additional long-term benefits, both at individual and health care system levels. With promising clinical outcome benefits, it may add economic value. However, the scale of these outcome benefits needs to be evaluated in large randomized trials. With the currently available outcomes data, it has been suggested that the price should be reduced to $\$ 4250$. (around 70\% of its current value) for PCSK9 inhibitors to be cost-effective. ${ }^{25}$

Despite the promising potential advantages of PCSK9 that have been addressed so far, there is one important limitation identified. The clinical response among patients with familial hypercholesterolemia varies widely and depends mainly on if patient is heterozygous or homozygous for the disease. ${ }^{26}$ Patients with a homozygous mutation for familiar hypercholesterolemia had a substantially reduced response to PCSK9, compared with patients who were heterozygous. ${ }^{26}$

One of the main drawbacks regarding literature supporting PCSK9 inhibitors is the paucity of longterm and large-scale clinical outcomes data. Largescale trials with long-term follow-up that are adequately powered are needed to address actual impact on clinical outcomes. Until then, its exact role for addressing residual ASCVD risk remains unclear. ${ }^{27}$

The expert panel of National Lipid Association has recently published a guideline document with recommendations on suggested indications for PCSK9 inhibitors. ${ }^{28}$ National Lipid Association recommends the use of PCSK-9 inhibitors to reduce the level of LDL-C in following conditions (Table 4):

\section{(1) Atherosclerotic Cardiovascular Disease}

a. Stable ASCVD patients with additional risk factors and LDL-C $\geq 70 \mathrm{mg} / \mathrm{dL}$ or non-HDL-C $\geq 100 \mathrm{mg} / \mathrm{dL}$, despite maximum tolerated statin dose with or without ezetimibe,

b. Progressive ASCVD patients with LDL-C $\geq 70 \mathrm{mg} / \mathrm{dL}$ or non-HDL-C $\geq 100 \mathrm{mg} / \mathrm{dL}$, despite maximum tolerated statin dose with or without ezetimibe.

(2) Phenotypic Familial Hypercholestrolemia (FH)

a. Phenotypic FH patients aged 40 years to 79 years with baseline LDL-C $\geq 190 \mathrm{mg} / \mathrm{dL}$ and 
Table 4. Indications for Treatment with PCSK9 Inhibitors

\begin{tabular}{ll}
\hline Disease States & Patients with ASCVD, HoFH, HeFH. \\
\hline $\begin{array}{l}\text { Prerequisite for } \\
\text { treatment }\end{array}$ & Decision to start PCSK9 inhibitor would depend on achieving specific target LDL-C levels in \\
& various conditions that increase ASCVD risk or in presence of ASCVD. \\
& PCSK9 inhibitors are started in addition to maximum tolerated statin with or without ezetimibe. \\
& PCSK9 inhibitors are started in statin-intolerant patients for achieving specific target LDL-C \\
& levels in various conditions that increase ASCVD risk or in presence of ASCVD. \\
& Stable with risk factors and in progressive LDL-C $\geq 70 \mathrm{mg} / \mathrm{dL}$ or non-HDL-C $\geq 100 \mathrm{mg} / \mathrm{dL}$. \\
Lipid levels in ACVD for & Age 40 years to 79 years and controlled ASCVD risk factors, with baseline LDL-C $\geq 190 \mathrm{mg} /$ \\
treatment & dL, and LDL-C $\geq 100 \mathrm{mg} / \mathrm{dL}$ or non-HDL-C $\geq 130 \mathrm{mg} / \mathrm{dL}$ with statin \pm ezetimibe. \\
$\begin{array}{l}\text { Lipid levels in HeFH for } \\
\text { treatment }\end{array}$ & Age 40 to $79 \mathrm{years}$ and uncontrolled ASVCD risk factors, baseline LDL-C $\geq 190 \mathrm{mg} / \mathrm{dL}$, and \\
& Age 18 to $39 \mathrm{years}$ with uncontrolled ASVCD risk factors, baseline LDL-C $\geq 190 \mathrm{mg} / \mathrm{dL}$, and \\
& LDL-C $\geq 100 \mathrm{mg} / \mathrm{dL}$ or non-HDL-C $\geq 130 \mathrm{mg} / \mathrm{dL}$ with statin \pm ezetimibe. \\
LDL-C $\geq 70 \mathrm{mg} / \mathrm{dL}$ or non-HDL-C $\geq 100 \mathrm{mg} / \mathrm{dL}$ with statin \pm ezetimibe. \\
$\begin{array}{l}\text { Lipid levels in HoFH for } \\
\text { treatment }\end{array}$
\end{tabular}

PCSK9, proprotein convertase subtilisin/kexin type 9; LDL-C, low-density lipoprotein cholesterol; HDL-C, high-density lipoprotein cholesterol; HoFH, homozygous familial hypercholesterolemia; HeFH, heterozygous familial hypercholesterolemia; ASCVD, Atherosclerotic cardiovascular disease.

with other ASCVD risk factors that are controlled and with no additional high-risk markers, but with LDL-C $\geq 100 \mathrm{mg} / \mathrm{dL}$ or nonHDL-C $\geq 130 \mathrm{mg} / \mathrm{dL}$, despite maximum tolerated statin dose with or without ezetimibe,

b. Phenotypic $\mathrm{FH}$ patients aged 40 years to 79 years with baseline LDL-C $\geq 190 \mathrm{mg} / \mathrm{dL}$ and with other uncontrolled ASCVD risk factors or with additional high-risk markers or genetic confirmation of $\mathrm{FH}$ and with LDL-C $\geq 70 \mathrm{mg} / \mathrm{dL}$ or non-HDL-C $\geq 100$ $\mathrm{mg} / \mathrm{dL}$, despite maximum tolerated statin dose with or without ezetimibe,

c. Phenotypic FH patients aged 18 years to 39 years with baseline LDL-C $\geq 190 \mathrm{mg} / \mathrm{dL}$ and with other uncontrolled ASCVD risk factors or with additional high-risk markers or genetic confirmation of $\mathrm{FH}$ and with LDL-C $\geq 100 \mathrm{mg} / \mathrm{dL}$ or non-HDL-C $\geq 130$ $\mathrm{mg} / \mathrm{dL}$, despite maximum tolerated statin dose with or without ezetimibe,

d. Homozygous FH patients with either of unknown genotype or those known to be LDL-C receptor defective and with LDL-C $\geq 70 \mathrm{mg} / \mathrm{dL}$ or non-HDL-C $\geq 100 \mathrm{mg} / \mathrm{dL}$, despite maximum tolerated statin dose with or without ezetimibe.

(3) Statin Intolerance

a. High-risk patients who require additional cholesterol-lowering therapies despite using adequate doses of other nonstatin lipid-lowering drugs.

\section{Summary}

PCSK-9 offers an option for additional LDL-C reduction beyond that that could be achieved by conventional lipid-lowering strategies to achieve recommended LDL-C goals in patients with high risk of ASCVD events. It is also recommended in patients for primary and secondary prevention of ASCVD in selected group of patients. PCSK-9 inhibitors are fairly well tolerated. The cost of medication is currently an issue and would require third party insurance support for a wider acceptance of this medication.

To see this article online, please go to: http://jabfm.org/content/ 31/4/628.full.

\section{References}

1. Catapano AL, Graham I, De Backer G, et al. 2016 ESC/EAS guidelines for the management of dyslipidaemias: the Task Force for the Management of Dyslipidaemias of the European Society of Cardiology (ESC) and European Atherosclerosis Society (EAS) Developed with the special contribution of the European Association for Cardiovascular Prevention \& Rehabilitation (EACPR). Atherosclerosis 2016; 253:281-344.

2. Baigent C, Blackwell L, Emberson J, et al. Efficacy and safety of more intensive lowering of LDL cholesterol: a meta-analysis of data from 170,000 participants in 26 randomised trials. Lancet 2010;376:1670-81. 
3. Stancu C, Sima A. Statins: mechanism of action and effects. J Cell Mol Med 2001;5:378-87.

4. Robinson JG. Management of familial hypercholesterolemia: a review of the recommendations from the National Lipid Association Expert Panel on Familial Hypercholesterolemia. J Manag Care Pharm 2013; 19:139-49.

5. Stone NJ, Robinson JG, Lichtenstein AH, et al. 2013 ACC/AHA guideline on the treatment of blood cholesterol to reduce atherosclerotic cardiovascular risk in adults: a report of the American College of Cardiology/American Heart Association Task Force on Practice Guidelines. J Am Coll Cardiol 2014;63: 2889-934.

6. Seidah NG, Awan Z, Chretien M, Mbikay M. PCSK9: a key modulator of cardiovascular health. Circ Res 2014;114:1022-36.

7. Lagace TA, Curtis DE, Garuti R, et al. Secreted PCSK9 decreases the number of LDL receptors in hepatocytes and in livers of parabiotic mice. J Clin Invest 2006;116:2995-3005.

8. Seidah NG, Benjannet S, Wickham L, et al. The secretory proprotein convertase neural apoptosisregulated convertase 1 (NARC-1): liver regeneration and neuronal differentiation. Proc Natl Acad Sci U S A 2003;100:928-33.

9. Abifadel M, Varret M, Rabes JP, et al. Mutations in PCSK9 cause autosomal dominant hypercholesterolemia. Nat Genet 2003;34:154-6.

10. Cohen JC, Boerwinkle E, Mosley TH, Jr., Hobbs $\mathrm{HH}$. Sequence variations in PCSK9, low LDL, and protection against coronary heart disease. N Engl J Med 2006;354:1264-72.

11. Preiss D, Mafham M. PCSK9 inhibition: the dawn of a new age in cholesterol lowering? Diabetologia 2017;60:381-9.

12. Zhang XL, Zhu QQ, Zhu L, et al. Safety and efficacy of anti-PCSK9 antibodies: a meta-analysis of 25 randomized, controlled trials. BMC Med 2015;13:123.

13. Sattar N, Preiss D, Robinson JG, et al. Lipid-lowering efficacy of the PCSK9 inhibitor evolocumab (AMG 145) in patients with type 2 diabetes: a metaanalysis of individual patient data. Lancet Diabetes Endocrinol 2016;4:403-10.

14. Raal FJ, Giugliano RP, Sabatine MS, et al. Reduction in lipoprotein(a) with PCSK9 monoclonal antibody evolocumab (AMG 145): a pooled analysis of more than 1,300 patients in 4 phase II trials. J Am Coll Cardiol 2014;63:1278-88.

15. Clarke R, Peden JF, Hopewell JC, et al. Genetic variants associated with $\mathrm{Lp}$ (a) lipoprotein level and coronary disease. N Engl J Med 2009;361:2518-28.

16. Robinson JG, Farnier M, Krempf M, et al. Efficacy and safety of alirocumab in reducing lipids and cardiovascular events. N Engl J Med 2015;372:1489-99.
17. Sabatine MS, Giugliano RP, Wiviott SD, et al. Efficacy and safety of evolocumab in reducing lipids and cardiovascular events. N Engl J Med 2015;372:1500-9.

18. Sabatine MS, Giugliano RP, Keech AC, et al. Evolocumab and clinical outcomes in patients with cardiovascular disease. N Engl J Med 2017;376:1713-22.

19. Nicholls SJ, Puri R, Anderson T, et al. Effect of evolocumab on progression of coronary disease in statin-treated patients: the GLAGOV randomized clinical trial. JAMA 2016;316:2373-84.

20. Schwartz GG, Bessac L, Berdan LG, et al. Effect of alirocumab, a monoclonal antibody to PCSK9, on long-term cardiovascular outcomes following acute coronary syndromes: rationale and design of the ODYSSEY outcomes trial. Am Heart J 2014;168: 682-9.

21. Sabatine MS, Giugliano RP, Keech AC, et al. Evolocumab and clinical outcomes in patients with cardiovascular disease. N Engl J Med 2017;376:17131722 .

22. Giugliano RP, Mach F, Zavitz K, et al. Design and rationale of the EBBINGHAUS trial: a phase 3, double-blind, placebo-controlled, multicenter study to assess the effect of evolocumab on cognitive function in patients with clinically evident cardiovascular disease and receiving statin background lipid-lowering therapy-A cognitive study of patients enrolled in the FOURIER trial. Clin Cardiol 2017;40:59-65.

23. National Clinical Guideline Centre. Lipid modification: cardiovascular risk assessment and the modification of blood lipids for the primary and secondary prevention of cardiovascular disease. London: $\mathrm{Na}-$ tional Institute for Health and Clinical Excellence; 2014.

24. Naci H, Brugts J, Ades T. Comparative tolerability and harms of individual statins: a study-level network meta-analysis of 246955 participants from 135 randomized, controlled trials. Circ Cardiovasc Qual Outcomes 2013;6:390-9.

25. Arrieta A, Page TF, Veledar E, Nasir K. Economic evaluation of PCSK9 inhibitors in reducing cardiovascular risk from health system and private payer perspectives. PLoS One. 2017;12:e0169761.

26. Raal FJ, Stein EA, Dufour R, et al. PCSK9 inhibition with evolocumab (AMG 145) in heterozygous familial hypercholesterolaemia (RUTHERFORD-2): a randomised, double-blind, placebo-controlled trial. Lancet 2015;385:331-40.

27. Mazhar F, Haider N. Proprotein convertase subtilisin/kexin type 9 enzyme inhibitors: An emerging new therapeutic option for the treatment of dyslipidemia. J Pharmacol Pharmacother 2016;7:190-3.

28. Orringer CE, Jacobson TA, Saseen JJ, et al. Update on the use of PCSK9 inhibitors in adults: recommendations from an expert panel of the National Lipid Association. J Clin Lipidol 2017;11:880-90. 